FORMATION Formation emploi

Revue française de sciences sociales

96 | octobre-décembre 2006

Quand la qualification fait débat(s)

\title{
Des aides-éducateurs « sans qualités » : cas limites ou révélateurs?
}

Educational assistants "without qualities": extreme or instructive cases?

Hilfslehrer „ohne Eigenschaften“: Grenzfall oder Indiz?

\section{Gérard Boudesseul}

\section{(2) OpenEdition}

\section{Journals}

Édition électronique

URL : http://journals.openedition.org/formationemploi/2528

DOI : 10.4000/formationemploi.2528

ISSN : 2107-0946

Éditeur

La Documentation française

Édition imprimée

Date de publication : 1 octobre 2006

Pagination : $39-51$

ISSN : 0759-6340

Référence électronique

Gérard Boudesseul, «Des aides-éducateurs « sans qualités » : cas limites ou révélateurs ? », Formation emploi [En ligne], 96 | octobre-décembre 2006, mis en ligne le 14 décembre 2009, consulté le 30 octobre 2020. URL : http://journals.openedition.org/formationemploi/2528 ; DOI : https://doi.org/ 10.4000/formationemploi.2528

(c) Tous droits réservés 
ordre que pour l'ensemble des Emplois-Jeunes (Cadet 2005b). Peut-on en conclure une efficacité de l'employeur à réduire la période de chômage de ces jeunes? Ou bien les jeunes en question auraient-ils vraisemblablement trouvé un emploi sans l'aide de cette mesure ? On touche ici à l'évaluation différentielle des politiques d'emplois aidés au regard des autres politiques d'emploi.

Or le satisfecit quantitatif global quant aux sorties du dispositif avec un emploi demande à être nuancé lorsqu'on entre dans le détail des parcours. Les sorties heureuses sont pour la plupart dues à la réussite aux concours externes communs à tout candidat désireux d'entrer dans la Fonction publique, plus qu'à un réel accompagnement des personnes par les Rectorats. En d'autres termes, l'Éducation nationale ne peut guère fournir la preuve d'une efficacité spécifique d'un accompagnement pourtant prévu dans le programme initial : ceux qui s'en sont le plus vite sortis sont aussi ceux qui ont le plus bénéficié des formations et ceux qui étaient déjà les plus diplômés (ibid.). On est donc fondé à voir ici un curieux paradoxe, pour une institution qui détient pourtant deux avantages comparatifs sur tout autre employeur: la formation n'est pas seulement une exigence pour ses salariés, elle est aussi l'objet de son activité principale. De plus, les procédures d'accompagnement, de conseil pédagogique, de tutorat y sont développées de longue date et sont, pour certaines, institutionnalisées (conseillers pédagogiques, maîtres de stage, directeurs de mémoires...) et connues des salariés d'un établissement quelconque, celui-là même dans lequel un aideéducateur peut, daventure, être affecté.

A fortiori, ces difficultés amènent à s'interroger sur les efforts d'accompagnement nécessaires à toute politique d'emplois aidés, lorsque les employeurs ne disposent pas de tels avantages comparatifs en matière de formation. Si l'on admet qu'un volet insertion demeure nécessaire afin de ne pas s'en remettre « en aveugle » à des ajustements de marché, on peut se demander s'il suffit de prescrire par voie réglementaire les conditions d'insertion sans s'assurer que les conditions organisationnelles s'y prêtent, en particulier dans les modalités de partage des tâches, et faute de pouvoir anticiper sur les interactions en situation de travail. Une originalité de ce dispositif étant de se présenter comme assez peu prescriptif, en faisant appel à l'émergence de besoins non satisfaits, on se demandera si le fait de renvoyer aux bénéficiaires des contrats la charge de la preuve de leur utilité aura contribué à résoudre ou non ces difficultés.

Il est vrai que le devenir des anciens Emplois-Jeunes est aussi fréquemment abordé sous l'angle des compétences qu'ils auront éventuellement accumulées pendant la période contractuelle pouvant aller jusqu'à cinq ans. Conformément au projet porté par le programme « Nouveaux Services, Emplois-Jeunes », ces compétences sont associées, sinon à l'émergence de nouveaux besoins, du moins à la mise en visibilité d'activités jusque-là peu formalisées, périphériques ou partiellement pourvues (Piot 2001, 2004).

La première partie sera donc consacrée à quelques spécificités du contexte de l'Éducation nationale, avant d'aborder dans les quatre parties suivantes les obstacles possibles à la construction d'une compétence chez les aides-éducateurs.

\section{L'ÉDUCATION : UN MILIEU PEU FAVORABLE AUX TRANSITIONS PROFESSIONNELLES ?}

La probabilité de trouver un emploi rapidement est connue grâce à des enquêtes quantitatives; elle concerne environ six à sept (Divay, 2003) et sept (Cadet, 2005a) jeunes ayant quitté le dispositif pour dix emplois. Dans l'Éducation nationale, cette proportion est respectée ; ainsi, en 2004, $65 \%$ des aideséducateurs occupaient un emploi cinq mois après leur sortie du dispositif. Toutefois, on observe aussi que parmi ces derniers, la moitié sont devenus fonctionnaires (soit $32 \%$ au total) (ibid.). Les enseignants sont les plus nombreux, soit $21 \%$ de l'ensemble des sortants selon une autre source (Cadet, 2003). C'est dire que la réussite à un concours de la Fonction publique est une des voies principales d'accès à un emploi durable. C'est dire aussi que ce modèle de réussite est fortement prégnant, au-delà des trajectoires réelles. Or, les épreuves de concours, tant dans leur contenu que dans les modalités de passation, laissent peu de place à l'expérience professionnelle informelle et diverse issue des multiples situations de travail. Les critères du savoir académique prévalent encore largement, en dépit de l'introduction d'épreuves «sur dossier » et d'un «troisième concours » dont les limites ont déjà été tracées (Cadet, 2005a). Certes, il s'agit d'un concours spécifique, ouvert à présent aux 
surveillants ayant trois ans d'ancienneté, mais il a bénéficié à $1,6 \%$ des admis au concours externe en 2004, contre $40 \%$ pour les postes de Gardiens de la paix proposés aux Adjoints de sécurité au ministère de l'Intérieur, outre une procédure de tutorat et de Validation des acquis de l'expérience (Debost, 2000).

Certains auteurs mentionnent du reste combien la notion de compétence s'est construite par opposition à la logique de transmission scolaire classique des connaissances; cette dernière restant figée sur une logique analogue aux standards de qualification, plus que sur « une individualisation de la relation salariale qui s'accompagne d'un effacement des repères collectifs. Chaque salarié détiendrait alors la responsabilité de la construction de sa qualification » (Pagnani, 2004, p. 131). Cette logique aura vraisemblablement incité les aides-éducateurs à privilégier la durée du contrat plus que son contenu, comme le suggérait, du reste, avec enthousiasme, la ministre initiatrice du programme ( «À cet âge-là, cinq ans, c'est une éternité »). Il aura bénéficié aux aides-éducateurs comme un temps de latence, un répit du même ordre que celui fourni par les emplois de surveillance. La notion de transition, au sens de progression graduelle et organisée vers un emploi ${ }^{3}$, ne s'appliquerait tout au plus qu'à trois aides-éducateurs sur dix, ceux qui ne sont pas entrés dans la Fonction publique.

Il est vrai que deux considérations peuvent être invoquées :

La première est que la construction des compétences porte l'empreinte spécifique du milieu d'accueil et de sa culture professionnelle, même s'il ne fait pas figure d'exception. Les personnels d'éducation et d'enseignement ont en commun une activité de service à forte composante d'interactivité relationnelle, mais aussi d'asymétrie en faveur du salarié, au regard du destinataire de ce service, le plus souvent en situation de dépendance (l'élève vis-à-vis de l'enseignant); ceci les apparente à d'autres professions telles que celles de la santé, physique et mentale, du travail social, et des soins aux personnes âgées, à des degrés divers. Si l'on fait ici abstraction du contenu

\footnotetext{
${ }^{3}$ « Processus singulier, long, complexe et organisé » correspondant à une « autonomisation progressive d'une période singulière de la vie [...] période d'enchevêtrement complexe d'états, de statuts, de différenciation des personnes, et surtout, période socialement organisée » (Rose, 1998, pp. 213-214).
}

très normatif de l'activité enseignante, lié en particulier au mandat de transmission, mandat dans lequel l'État est fortement impliqué en France, cette forte dose d'interactivité et d'asymétrie est pour beaucoup dans l'investissement des enseignants et des personnels d'éducation dans leur tâche. Les sociologies québécoise du «travail enseignant» et américaine de «l'écologie de l'éducation » ont fortement développé ces aspects (Tardif, 1999 ; Doyle, 1985). Dans notre cas de figure, les récits recueillis montrent l'importance de cette interactivité et de cette asymétrie dans les appréciations portées sur l'expérience. De plus, l'investissement est très marqué, comme l'illustrera le cas d'Aurore, qui est prête à se priver de repas pour «bien faire » son travail. Dans cette perspective, les rôles sont assez nettement établis, bien qu'une partie des tâches soient négociables, et trouvent de nombreux ressorts au quotidien, quelle que soit la pénibilité des tâches quotidiennes. Il est donc plausible que cette culture professionnelle ambiante ait freiné les projets à long terme des aides-éducateurs dans les établissements. On comprendrait qu'une partie de la demande de reconnaissance se transforme en une demande de pérennisation de l'existant (implicitement véhiculée par la revendication syndicale de pérennisation des emplois). Dans cette logique, la transition se serait en quelque sorte résumée à une installation dans le provisoire.

Une seconde considération peut aussi être invoquée pour justifier d'une difficile transition dans la construction progressive de compétence: la porosité croissante de cette culture professionnelle à l'égard des mutations générales de l'économie et du marché $\mathrm{du}$ travail affecterait immanquablement aussi la construction des qualités requises à un poste de travail (Lessard, 2000). C'est cette considération qui aura notre préférence.

Le récit de vie ( $c f$. encadré 1) d'une douzaine de jeunes aides-éducateurs montrera comment, lorsqu'un milieu d'accueil déjà déstabilisé par les mutations du public scolaire, des demandes des familles, et par la porosité évoquée, se prête mal à une reconnaissance de compétences nouvelles et spécifiques, faire miroiter comme atout principal un emploi d'une durée de cinq année a pu contribuer à construire des jeune salariés, et plus souvent des jeunes femmes, « sans qualités » (Sennett, 1998). Richard Sennett est en effet à l'origine de ce rapprochement entre les conditions de travail et le «caractère» des 
personnes ${ }^{4}$. L'expérience au travail a une incidence sur la représentation que la personne a de ses propres qualités, en particulier de sa capacité à résoudre des situations-problèmes de manière autonome, des valeurs dont elle pense se prévaloir et des ressources collectives qu'elle se sent en mesure de mobiliser.

Les définitions de la compétence sont fort nombreuses, et sont inégalement applicables dans le cas

${ }^{4}$ Le travail sans qualités, cité en référence, dont le titre original est The corrosion of Character. The personal Consequences of Work in the New Capitalism : "Le caractère est la valeur éthique que nous attachons à nos désirs et à nos relations avec les autres [...] Le caractère a des accointances particulières avec l'évolution à long terme de notre expérience émotionnelle. Il s'exprime par la loyauté et l'engagement mutuel, à travers la poursuite d'objectifs à long terme, ou encore par la pratique de la gratification différée au nom d'une fin plus lointaine. » pp. 10-11

Sennett fait l'hypothèse que la dépossession au travail rejaillit sur l'ensemble des représentations de la personne, en particulier dans la présentation de soi dans la sphère familiale. Nous nous limitons à la représentation de l'emploi et du travail, dans ce que Becker appelle « la perspective » : «Ensemble coordonné d'idées et d'actions qu'une personne utilise pour faire face à une situation problématique. » (Becker, Hughes, Geer, Strauss (1961) 1992, pp. 34-35) présent $^{5}$. L'accent est généralement mis sur la combinaison de ressources plurielles, la singularité des situations concrètes de travail, l'interactivité avec le collectif de travail, et enfin, trois aspects ayant trait à leur mise en visibilité : elles sont mobilisées, et donc repérables, dans l'action. Elles sont donc évaluables ex post sur des résultats obtenus, ce qui, finalement, tend à polariser l'affichage des qualités requises ex ante autour des savoir-être, plus que des savoirs ou des savoir-faire, même si ceux-ci ne sont pas absents (Bellier, 1998). Ici, quatre aspects de la compétence semblent affectés par la singularité du contexte et les interactions collectives locales.

\begin{abstract}
${ }^{5}$ Par exemple : «Combinaison de ressources mobilisées par l'individu dans une situation de travail. Elle résulte de la rencontre entre différents savoirs du salarié, d'un côté, et les moyens fournis par une situation de travail, de l'autre. » (Bouclet, Thomas 1999, p. 7) «La compétence permet d'agir et/ou de résoudre des problèmes professionnels de manière satisfaisante dans un contexte particulier en mobilisant diverses capacités de manière intégrée » (Bellier, 1999, p. 226).
\end{abstract}

\section{Encadré 1 \\ La méthode des récits de vie et son usage}

Un questionnaire $a$, en premier lieu, été soumis à un groupe témoin de soixante-dix aides-éducateurs respectant les proportions par sexe, âge et type d'affectation de l'ensemble des aides-éducateurs de la région d'observation. Une douzaine de récits biographiques ont ensuite été recueillis parmi ces enquêtés, comprenant aussi bien des parcours de réussite que d'échec. Ils ont ensuite été croisés avec des sources administratives de l'employeur émanant d'autres acteurs des établissements d'affectation, comme des enseignants en poste. Un entretien collectif a enfin réuni sept aides-éducateurs, un professeur des écoles et un professeur de collège. La méthode des récits de vie se justifie par sa capacité à restituer la logique d'ensemble d'un parcours, et particulièrement ce qu'il advient d'une formation et d'une expérience antérieures lorsque la personne se trouve confrontée à une situation hybride mêlant travail, projet de formation et d'emploi. La question traitée est moins celle de la représentativité statistique de leurs positions successives que de l'identification de cohérences locales et de parcours singuliers, dès lors que ceux-ci sont en eux-mêmes porteurs d'un sens global. Ce sens suffit, ad minima, à douter de la généralité du modèle dominant ou le plus fréquent, ici celui du succès quantitatif de l'insertion professionnelle des aides-éducateurs. Une difficulté survient lors de la restitution des matériaux, qui sont difficiles à synthétiser sans mutiler le sens des parcours : les contraintes éditoriales nous ont amené à citer des extraits en contrepoint des arguments, alors qu'au fil de la recherche, ce sont les récits entiers qui ont été émaillés de commentaires, lesquels, de récit en récit, ont conduit à ajuster les hypothèses sur le modèle de la "mosaïque » suggéré par Becker. Les titres des parties de l'article constituent la forme aboutie de ces hypothèses, jusqu'à plus ample information, dans un cheminement de recherche qui reste à dominante inductive. 
En premier lieu, l'accent est mis sur la pluralité des ressources à mobiliser et donc la nécessité d'opérations mentales autonomes permettant de les combiner, ce qui est particulièrement pertinent lorsque les tâches sont nouvelles ou peu formalisées. Toutefois, les aides-éducateurs ont été mis en demeure de démontrer ces qualités d'autonomie face à des tâches multiples, alors que le contexte quotidien des relations de travail les a plutôt mis dans une situation de dépendance. Les aides-éducateurs interrogés vont plus loin : ils estiment avoir été instrumentalisés.

En second lieu, cette mobilisation individuelle qu'on attend d'eux implique une appropriation d'objectifs partagés. Plus en amont, comme dans le cas d'activités de services relationnels, ou du service public, cette appropriation peut aller jusqu'à l'intériorisation de normes et de valeurs qui sont considérées comme des conditions d'exercice des professions de l'éducation. Les aides-éducateurs interrogés, désireux de s'intégrer à l'institution, ont fréquemment manifesté, dans les entretiens, ce partage de normes et de valeurs communes. Toutefois, l'appropriation des normes laisse peu de place à une distance critique puisque les contraintes organisationnelles tendent à être objectivées pour elles-mêmes et non en référence à des objectifs plus larges ou des valeurs. Elles semblent si évidentes qu'il n'est plus guère envisageable de s'y soustraire.

En troisième lieu, une compétence n'existe que dans la mesure où elle est évaluable et transférable. Des opérations complexes sont mobilisées en amont et au moment de la situation de travail, par exemple en faisant appel à des qualités de "savoir-être »; dès lors, l'évaluation est conditionnée par des résultats tangibles. Pour les aides-éducateurs interrogés, à qui on a demandé de faire la preuve de leur utilité, cette injonction est particulièrement source de tension; alors que sont déjà débattues chez les enseignants la possibilité même de calculer une valeur ajoutée ou une productivité de leur activité, et la nécessité de rendre des comptes. Les difficultés générales de l'évaluation prennent la forme d'un déficit de reconnaissance professionnelle, particulièrement visible dans les situations de conflits. Les divergences de vues sur les formes de savoir-être à mobiliser situent toujours les rapports de travail sur le registre des relations interpersonnelles et de la subjectivité.

En quatrième lieu, les rapports de travail sont moins perçus comme résultant d'une configuration profes- sionnelle et collective, que comme un arrangement local entre individualités dont certaines sont en situation d'exercer un pouvoir sur d'autres. Les aideséducateurs revendiquent alors diverses formes de reconnaissance, en particulier sur le plan de «l'utilité », de la sociabilité, voire de la nature humaine.

Nous allons développer, en les illustrant, ces quatre aspects qui pourraient expliquer la rareté relative de véritables transitions professionnelles, progressives et encadrées, dans l'Éducation nationale : la tendance à l'instrumentalisation des personnes, l'objectivation organisationnelle des contraintes, la subjectivisation des rapports de travail, et la revendication de reconnaissance extra-professionnelle.

\section{UNE INSTRUMENTALISATION DES PERSONNES PAR DÉLÉGATION}

Avec les aides-éducateurs et les assistants d'éducation, un aspect inédit apparaît, du fait même de l'inachèvement de leur insertion dans le système éducatif.

L'inachèvement de l'insertion des aides-éducateurs ne leur est pas spécifique. Il révèle l'inachèvement, sur le long terme, de la répartition des tâches dans les métiers de l'éducation. Pourtant, le fait nouveau tiendrait dans ce redéploiement de personnes par lequel une division des tâches nouvelle se superpose à une ancienne, sans toutefois s'y substituer. Du point de vue des nouveaux arrivants, cette configuration a des effets spécifiques: il n'y a pas de bureau des méthodes, pas d'expert des relations humaines qui imposent hiérarchiquement et unilatéralement une redistribution des tâches. La division du travail est elle-même en partie flexible, alors qu'un halo de tâches plus ou moins floues et inégalement reconnues entoure les fonctions plus étroitement codifiées de l'enseignement face à un groupe-classe. Les aideséducateurs sont donc arrivés dans une situation où la distribution des tâches n'était ni figée, ni totalement prescrite d'en haut. Il s'ensuit que leur affectation n'a pas pu suivre un principe courant d'un homme pour chaque place, et encore moins du «right man on the right place » (la bonne personne à la bonne place), mais plutôt d'une place à trouver pour chacun. Cette logique a été prévue par les initiateurs des EmploisJeunes au nom de l'émergence de gisements d'emplois et de nouveaux besoins méconnus. Les aides-éducateurs arrivent donc moins comme des 
supplétifs d'une organisation du travail en mal de rigidité que comme les facteurs d'identification de nouveaux besoins.

En fait, le flou entourant leurs attributions a favorisé une forme spécifique d'instrumentation des personnes : le dernier arrivé subit les conséquences des arrangements mis en place par ceux qui l'ont précédé, et qui sont détenteurs des attributions les plus formalisées. On pourrait parler d'une instrumentation par délégation au sein de la collectivité de travail, dans la mesure où les personnels titulaires des établissements scolaires disposent d'une autonomie non négligeable et croissante, surtout dans le second degré. La sociologie des enseignants a montré l'ambivalence du collectif de travail : d'un côté, une structure cellulaire comparable à un "panier à oeufs » favorise un certain individualisme, certains auteurs mentionnant une forme de "concurrence sans conflits », alors que ces derniers font traditionnellement l'objet d'une aversion dans le milieu. D'un autre côté, les incitations ou les injonctions à l'innovation et au travail collectif se traduisent fréquemment par une coopération de proximité dans laquelle les affinités jouent de manière significative (Barrère, 2002).

Constatant l'existence de dérives, des précisions réglementaires ont été apportées, interdisant en particulier le recours aux aides-éducateurs pour la prise en charge pédagogique autonome d'un groupe-classe. Pourtant, des arrangements locaux se sont instaurés en toute transgression : des cas sont apparus où des enseignants en poste ou des responsables d'établissements ont confié à des aides-éducateurs des demiclasses en responsabilité, voire des classes entières en cas de remplacement de titulaires absents.

Aurore est ainsi très absorbée, on pourrait dire immergée, dans son travail. Affectée sur quatre, puis l'année suivante, sur trois écoles, dans un rayon de vingt kilomètres, elle fait la navette plusieurs fois par semaine entre ses employeurs qui la sollicitent. Le plus souvent, elle prend en charge des demi-classes en informatique, pour lesquelles elle a confectionné des fiches pédagogiques à l'aide d'un logiciel. Elle fait aussi du soutien scolaire pour des élèves en difficulté ou du travail avec des groupes restreints : «Je garde les CE2 d'une classe de CE1-CE2 pendant que l'enseignant fait sport avec les autres. En général, elle m'a préparé un travail à faire. J'en surveille huit qui font le travail et dans la salle qui communique, à côté, les huit autres ont quelque chose à taper, donné par l'enseignant, ou bien qu'on a vu ensemble. ", (Aurore, 25 ans, Deug - diplôme d'études universitaires générales - psychologie, licence Sciences de l'éducation) Enfin, elle remplace parfois des enseignants absents, en toute illégalité. En tout, elle intervient sur les sept niveaux des écoles maternelles et primaires, ce qui n'arrive jamais à aucun enseignant titulaire. Pourtant, elle ne formule ni critique, ni réticence à exercer ces responsabilités, à la différence de nombreux aideséducateurs qui se plaignent d'être considérés comme des supplétifs ou des « bouche-trous ».

Rachel, quant à elle, semble dans l'impasse. Alors que l'avenant à son contrat mentionne qu'elle est spécifiquement affectée à un centre de malvoyants au sein d'un collège, elle est sollicitée pour des tâches de surveillance durant un quart de son emploi du temps. Certes, elle ne cherche pas spécifiquement dans cet emploi un travail auquel elle pourrait s'identifier: «Après trois mois de chômage, c'est un peu comme surveillant, ça me permettait d'avoir une formation à côté, de continuer ma formation, enfin, c'est ce que je croyais. » Mais elle exprime le sentiment d'être utilisée, non reconnue dans son travail : «Les enseignants, ils profitent qu'on soit là, point. Pour eux, je suis une secrétaire. » De plus, l'organisation de l'emploi du temps la bloque dans son projet de formation, car elle ne pourra pas suivre le stage de douze semaines réparties sur l'année de la licence professionnelle qu'elle prépare (Rachel, 29 ans, Deug Géographie, licence pendant le contrat, affectée en collège). Et Élise de renchérir :

" On est sans arrêt sollicités pour n'importe quoi, on est les petits soldats. Sur six Emplois-Jeunes, il y en a cinq qui ne font quasiment que de la surveillance. Ils ne peuvent pas remplir leur mission à cause de cela. On se substitue aux surveillants » (Élise, 23 ans, Deug Histoire, affectée en collège). Cette estimation, alors qu'elle est en situation de conflit avec la proviseur-adjointe, dépasse quelque peu les chiffrages nationaux, qui font état de $61 \%$ d'aides-éducateurs déclarant les tâches de surveillance en collège, et de $8 \%$ comme activité principale (Diederichs, 2001).

La spécificité d'Aurore, dans ces quatre extraits de récit, est que non seulement elle ne remet pas en cause les sollicitations dont elle est l'objet, mais de plus, elle y voit une concrétisation de ce qu'elle espère un jour faire, et qu'elle condense en fin d'entretien, avec une forte conviction: «Moi, ce que je voudrais, c'est avoir ma classe à moi. » Or, son impli- 
cation est telle qu'elle se prive des moyens et du temps nécessaires pour bénéficier pleinement de la journée hebdomadaire de préparation au concours qu'elle reçoit à l'IUFM (Institut universitaire de formation des maitres). Elle échoue trois ans de suite au concours, pour être admissible aux épreuves orales la quatrième année.

On peut donc parler d'une instrumentalisation par délégation, en ce sens que ni une structure hiérarchique pyramidale, ni un bureau d'expert n'imposent les attributions.

Ce mécanisme de délégation locale de tâches, considérées comme moins centrales ou moins prestigieuses que le face-à-face avec la classe, s'inscrit assez bien dans le schéma de "délégation du sale boulot » proposé par Hughes pour toute profession, du concierge au musicien, en passant par l'infirmier et l'enseignant (Hughes, 1996). La différence avec la relation entre le chirurgien et l'infirmier tient toutefois à son caractère non hiérarchique, exigeant donc un certain consensus, en particulier dans ce qu'il faut convenir de désigner comme un besoin nouveau.

\section{Une objectivation organisationnelle des contraintes}

Les contraintes matérielles, administratives et temporelles d'un établissement scolaire sont d'ordinaire assez lourdes. Pourtant, cette critique souvent présente chez les enseignants en poste sous la forme d'impossibilité à concilier des prescriptions d'origines multiples, n'est pas présente dans les récits des aides-éducateurs. Ils semblent au contraire les endosser plutôt rapidement.

Elles sont de deux ordres : celles qui sont partagées avec les titulaires en poste, et celles qui sont reportées sur les aides-éducateurs. Les règles partagées émanent de l'École en tant qu'institution, c'est-à-dire qu'elles reposent sur les valeurs et finalités communes aux missions de l'Éducation, aussi bien sur le plan de la transmission intergénérationnelle que de la formation de la citoyenneté républicaine. En revanche, les règles reportées sont le fruit de stratégies diverses, parfois congruentes avec un discours de la professionnalisation visant à redéfinir le « cœur de métier» des enseignants à l'appui d'une certaine expertise et d'un gain d'autonomie. À la différence des précédentes, l'origine de ces règles est moins perçue par les aides-éducateurs interrogés comme émanant de l'institution, mais plutôt de l'organisation éducative : détachées de leur finalité, elles s'imposent d'elles-mêmes sous le sceau de la nécessité fonctionnelle et de l'urgence. Paradoxalement, cette «emprise organisationnelle (Etzioni, 1993) est d'autant plus forte lorsque les motifs d'engagement dans le contrat d'aides-éducateurs demeurent ancrés dans les valeurs lointaines de l'institution. Les contraintes organisationnelles sont d'autant plus acceptées que l'espoir existe de passer un jour de "l'autre côté », c'est-à-dire de devenir soi-même enseignant. Le temps d'un purgatoire, en quelque sorte. Par exemple, Aurore est à ce point sollicitée qu'elle prend sur ses heures de sommeil et hâte ses repas, lorsqu'elle ne s'en prive pas, parce que les horaires des différentes écoles dans lesquelles elle travaille sont décalés : "Le vendredi, il faut que je mange vite, vite, parce que je dois aller à la troisième école. Les après-midi aussi sont décalés : on reprend à $13 \mathrm{~h} 30$ dans la première et à 12 h 50 dans la deuxième. Au début, je croyais qu'il y avait des raisons pédagogiques à cela. En fait, c'est uniquement en fonction de la tournée des bus ! »

La forme rationalisée qu'elle livre de cette tension est le manque de temps. Par un processus tout à fait analogue à celui évoqué par Sennett (1998), elle reporte ce registre de la contrainte temporelle sur la formation qu'elle reçoit à l'IUFM pour préparer le concours de professeur des Écoles : «Les cours sont bien mais on n'a pas assez de temps. C'est normal qu'il y ait un contrôle d'assiduité, mais des fois, on n'a pas le temps de souffler, même pour faire les préparations pour l'école ». Plus largement encore, elle tend à généraliser les contraintes quotidiennes de temps dans l'urgence pour elle de rompre avec son statut d'étudiante et de stabiliser sa conquête d'indépendance à l'égard de ses parents : "J'ai déjà échoué au concours l'an dernier, il faut que je me dépêche avant la fin du contrat. Après, je ne pourrai plus demander une inscription en première année d'IUFM. J'ai 25 ans, j'ai quitté mes parents à 20 ans, je me suis débrouillée toute seule en étant vendeuse à mi-temps. »

Elle accepte et se reconnaît dans cette division des tâches: "Le jeudi, en théorie, je prends les $C P$ en informatique, mais s'il $\boldsymbol{y}$ a une fête des mères, une fête des pères, un carnaval, une peinture à finir... c'est pour moi. L'instit sait que c'est son après-midi [...] Le vendredi, je suis en CE2-CM1, l'enseignante a 
droit à sa journée aussi. C'est un soulagement pour elle. À sa demande, on fait tel exercice, telle page. $»^{6}$

Dans cette logique, les contraintes cessent de faire partie d'un tout articulé autour de finalités, de ressources professionnelles, de règles communes, de droits et de devoirs collectifs. Elles sont décontextualisées, déprofessionnalisées, et tirent l'essentiel de leur légitimité de l'objectivité apparente de la raison organisationnelle. Si les rapports interpersonnels n'en sont pas absents, le coût (en termes de tension ou de conflit possible) des injonctions en situation de face-à-face s'en trouve réduit. D'autant qu'Aurore n'a pas seulement à répondre aux attentes d'un groupe d'enseignants dans une école, mais à concilier les contraintes en partie concurrentes des trois, voire quatre établissements dans lesquels elle est affectée. La difficulté à préserver une distance critique à l'égard des contraintes et à faire preuve de réflexivité à l'égard de ses propres choix entrave la logique d'apprentissage. Or, la réflexivité figure fréquemment au rang des dix premières compétences attendues des enseignants dans une perspective de professionnalisation (Perrenoud, 1999).

La réaction n'est pourtant pas toujours celle de l'adhésion: elle peut prendre la forme d'un conflit pour le rejet d'une partie des tâches assignées, qui font l'objet d'une hiérarchisation locale, ou de la défense d'un périmètre d'engagement.

\section{Une subjectivisation des rapports de travail exacerbée par les conflits}

$\mathrm{Si}$, dans certains cas, les conflits ont pu bénéficier d'une couverture syndicale, et de ce fait trouver une traduction dans un nouveau dispositif local de prescription des tâches, dans d'autres, ils demeurent sur un registre de forte subjectivité des rapports quotidiens de travail, en même temps qu'ils les révèlent.

Il est vrai que le milieu professionnel comporte une dimension subjective de par la nature de la relation

\footnotetext{
${ }^{6}$ Une analyse sémantique affinée du récit révèle un usage asymétrique des adjectifs et des pronoms possessifs : ce qui est «à moi » est objectivé par des formes lexicales impersonnelles («en théorie... s'il y a... c'est...»), et ce qui est « son » après-midi ou «sa » demi-journée est légitimé par les catégories collectives du « droit », l'équité de « aussi », et le « soulagement».
}

pédagogique, avec une accentuation de demandes adressées aux enseignants pour qu'ils répondent à des besoins individualisés. La subjectivisation des rapports de travail a donc une légitimité professionnelle, comme dans d'autres professions de services relationnels à forte composante d'interactivité et d'asymétrie vis-à-vis de l'usager.

Toutefois, l'objectivation organisationnelle des contraintes tend à réduire les marges d'autonomie des aides-éducateurs, vus comme des acteurs insérés dans un collectif de travail et des rapports de pouvoir (Sainsaulieu, 1973). Cette perte d'autonomie réduit la possibilité de se référer à une identité collective au travail, et confine les aides-éducateurs dans un espace d'identification plus restreint, désigné « modèle affinitaire » par Sainsaulieu.

Le risque est alors de se trouver enfermé dans un conflit de personnes où le face-à-face devient décisif, accentuant l'anxiété produite par la culpabilité face aux injonctions et l'incertitude quant à l'après-contrat.

Ainsi, Perrine a eu maille à partir avec la commission rectorale de suivi des projets de formation qui a refusé de valider son projet: "C'est ridicule! Et c'est facile de culpabiliser les gens alors que derrière tout cela il n'y a qu'une question de budget. » (Perrine, 29 ans, Deug Psychologie, travaille dans deux écoles). Elle dresse un bilan désabusé de ces cinq années: "Cinq ans d'investissement dans les écoles, cinq ans d'investissement dans des projets [...], et tout ceci pour que la correspondante de la cellule Emplois-Jeunes me dise que je n'ai pas réussi à m'insérer! J'ai bientôt trente ans, à quoi m'ont servi ces cinq années payées au SMIC (salaire minimum interprofessionnel de croissance), puisqu'au bout il n'y a rien? Je suis révoltée, écœurée par tout ce système, par tout le "bla-bla" du départ, nous faisant espérer des formations qui aboutiraient à un travail, alors qu'il n'en est rien. »

Tanguy a lui aussi eu l'expérience du conflit, avec le Proviseur du lycée professionnel où il est affecté : «Pendant un an et demi, deux ans, elle m'a poussé à bout. » (Tanguy, 27 ans, DUT - diplôme universitaire de technologie - gestion, licence Sciences de l'éducation). Et d'énumérer une longue liste de griefs, parmi lesquels le mot « reproche » apparaît sept fois, dont la modeste teneur importe moins que le registre personnalisé qui est utilisé : « Madame Le Proviseur me fait savoir qu'en tant que proviseur elle a un statut mais 
que les professeurs n'ont qu'un grade [...] Ensuite, elle m'indique que je n'ai rien appris en un an, et que pourtant, elle m'a appris comment on présente un document dans ce lycée, à dire bonjour le matin, à m'excuser avant d'interrompre les gens. Elle me reproche de faire preuve de mauvaise volonté, de bâcler le travail qui ne m'intéresse pas. Elle conclut sur le fait qu'elle sera "obligée de me renvoyer à la vie active" si la situation ne s'améliore pas. " $\mathrm{Si}$ Tanguy trouve un soutien syndical qui aboutit à un compromis, les tensions et conflits demeurent le plus souvent sur ce registre du face-à-face individualisé. L'émotion dans laquelle ce récit plonge Tanguy, alors que l'épisode est clos depuis près d'un an, montre qu'il en demeure durablement affecté. La difficulté de trouver un terrain d'entente tient au tour infantilisant que semble avoir pris le conflit, mais aussi qu'une partie des griefs ne porte pas seulement sur des tâches mais aussi sur des attitudes générales qui illustrent la difficulté à prendre en compte le registre des « savoir-être ».

\section{Une revendication réduite à une demande de reconnaissance}

Une esquive possible est alors de s'accommoder des interactions conflictuelles grâce à des considérations générales et pacifiantes sur les rapports humains et les traits de caractère que l'on rencontre dans l'ensemble de la société.

L'objectivation organisationnelle des contraintes accentue d'une part un certain conformisme à l'égard $\mathrm{du}$ milieu d'accueil, sans pour autant que les aideséducateurs bénéficient des prérogatives et des rétributions matérielles et symboliques des titulaires. D'autre part, la tendance à la subjectivisation des acteurs professionnels en présence réduit leur chance de pouvoir s'appuyer sur une identité propre. Le champ des revendications possibles se trouve alors restreint, soit à des aménagements réglementaires, soit à des considérations générales en termes de demande de reconnaissance et d'intégration.

Sylvie passe d'abord rapidement sur le fait que « Oui, les relations, ça se passe bien, moi et ma collègue, on est respectées, disons, à $60 \%$ \%. Interrogée sur les $40 \%$ restants: "C'est des remarques du genre "Tenez, vous voulez aller me chercher un café, ou corriger des copies, j'ai pas envie de faire la récré, vous allez la faire...", ou encore on est considérée comme la secrétaire particulière du directeur, il faut taper des lettres, coller des enveloppes..." (Sylvie, 29 ans, Deug AES - Administration économique et sociale -, affectée sur une école)

Les tensions, puis le conflit, durent deux ans, à la suite de quoi un courrier est adressé à l'inspecteur de circonscription, qui semble apaiser les tensions. Mais aujourd'hui encore, Sylvie ne présente pas cet épisode comme un conflit de relations professionnelles : "C'est un problème de rapports humains. Si je contredis le directeur, il avouera jamais, il est têtu, çà il est têtu. » Sylvie revient plusieurs fois sur cette rationalisation en termes de rapports humains. C'est en habillant ce registre de normes déontologiques qu'elle va accepter certaines tâches pédagogiques, alors même que pour des raisons de calendrier, cela va la pénaliser pour passer un concours blanc organisé par l'IUFM : "Normalement, on ne devrait pas faire de classes découvertes, parce qu'on n'est pas censés travailler entre 21 h et 6 h. Bon, je vais le faire quand même, parce que j'ai une conscience professionnelle, je tiens à le dire, même si quand on reviendra, je serai crevée et ce sera à deux jours des concours blancs. »

Pour Perrine, la mise en demeure de démontrer son utilité pour justifier l'éventuelle pérennisation de l'emploi, sous le statut d'assistant d'éducation, a eu un effet analogue à celui de la demande de preuve permanente de compétences dans les entreprises : les qualités professionnelles ne sont pas acquises, elles demandent à être quotidiennement démontrées. Perrine y échoue.

Il est vrai que son expérience de l'école et du marché du travail lui laissent peu de ressources. De nombreux choix engageant sa carrière scolaire et professionnelle ont été pris par d'autres : orientée en section G (secrétariat) par défaut, alors qu'elle avait un goût pour la littérature, échouant au concours d'assistante sociale pendant la classe de Terminale, s'inscrivant en Psychologie à l'Université sur le conseil d'un conseiller d'orientation, mais échouant aux épreuves de Physiologie, elle abandonne à la fin de la deuxième année, qu'elle finit pourtant par obtenir pendant son contrat d'aide-éducatrice. Pour celui-ci, elle impute le succès de son recrutement au fait «qu'ils avaient dî faire une erreur ». Ensuite, persistant dans son intérêt pour le travail social, la préparation au concours d'éducateur spécialisé lui est refusée. Son expérience 
du marché du travail ne lui apporte pas plus de confiance en elle : elle peine d'abord à trouver un emploi de vendeuse "parce qu'ils demandaient une expérience », emploi qu'elle finit par obtenir mais dont elle est licenciée, parce que «c'était une période d'essai, je ne correspondais pas ».

Le point commun de ces multiples bifurcations est que Perrine est dépossédée des choix qui ont orienté sa carrière. Ce sentiment de dépossession semble rejaillir sur sa manière d'aborder l'activité d'aideéducatrice: elle refuse défensivement de prendre seule des classes en charge, et dénonce l'insuffisance de formation et d'expérience valorisables sur le marché du travail. Les seuls points qu'elle fait valoir positivement sont de l'ordre de la sociabilité et de la reconnaissance qu'elle revendique. Vis-à-vis des élèves tout d'abord: "Avec nous, il y a moins de distance, l'enfant pourra vous confier des choses comme à une grande sœur. Mais surtout, la différence, c'est qu'on ne leur met pas de notes, on ne les juge pas. » Vis-à-vis des enseignants : «Spontanément, ils mettent une distance [...] ça reste très travail, les élèves, tout çà... au début, ils ont eu du mal à m'intégrer. » Elle y oppose la sociabilité plus affinitaire qu'elle a rencontrée à La Poste comme contractuelle : «Entre collègues, on parlait de tout, même du privé. » On peut se demander si ce repli sur des revendications de l'ordre de la sociabilité extraprofessionnelle n'exprime pas en creux le fait qu'elle a été dépossédée non seulement de la possibilité de faire des choix importants, mais des qualités qui seraient nécessaires pour y parvenir. On retrouve ici l'approche de Sennett, lorsqu'il écrit de Rico, consultant, fils d'immigré italien soumis aux incertitudes de la flexibilité " qu'il n'a pas grand chose à offrir en guise de modèle de rôle » (Sennett 1998, p. 30).

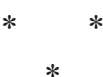

Dans l'ensemble, ces quatre aspects pourraient contribuer à expliquer pourquoi l'Éducation nationale se prête assez mal à l'organisation d'une transition professionnelle dans le cas des aides-éducateurs, en dehors des incitations à passer les concours. Dans un cadre où les contraintes organisationnelles sont fortes, les arrangements locaux semblent plutôt fluctuants et les collectifs inégalement réceptifs à l'irruption de nouveaux venus.
Nous avons vu, en premier lieu, que l'instrumentalisation, ressentie ou avérée, place les personnes qui en sont l'objet en situation de dépendance, peu propice à la conquête d'une autonomie en situation de choix professionnels : face à la multiplication des problèmes à résoudre ou des missions à remplir, ils risquent au contraire de se trouver paralysés par des injonctions paradoxales, surtout si cette dépendance est peu formalisée dans les interactions de travail.

En second lieu, les valeurs par lesquelles les personnes se rendent estimables aux yeux de leur entourage, dans le travail mais aussi hors travail, sont reportées sur un long terme associé à l'espoir d'une réussite au concours, alors que les contraintes organisationnelles, en particulier temporelles, s'imposent avec une légitimité propre.

En troisième lieu, le collectif est une ressource de la coopération, de la négociation, mais aussi de la régulation des relations de travail (Reynaud, 1979). Il se trouve fragilisé par une tendance à l'individualisation dans un milieu déjà favorable à celle-ci, et à la subjectivisation des conflits.

En conclusion, les situations incertaines dans lesquelles se sont trouvés certains aides-éducateurs à l'issue de leur contrat n'oblitèrent ni le caractère novateur d'un dispositif, ni le mérite de ceux, les plus nombreux, qui ont trouvé une issue dès les premiers mois qui ont suivi. Les difficultés évoquées invitent à nuancer certains concepts, tels que ceux de transition professionnelle et de compétence, mais aussi à réfléchir aux conditions dans lesquelles peuvent être déployées les mesures d'accompagnement de toute politique d'emplois aidés.

Sur le plan conceptuel, la notion de compétence atteint ici ses limites. La logique de la qualification a eu ce mérite de rendre compte de processus ambivalents comme celui des effets «déqualifiants» et "surqualifiants" de l'introduction de machines, rappelant de loin le principe schumpetérien de la destruction créatrice. Ici, la logique de la compétence ne permet guère de lire de la même manière les effets ambivalents des "machines organisationnelles », plaçant les acteurs sous ce que d'aucuns ont appelé l'emprise organisationnelle: comme l'a montré Etzioni (1993), les différentes voies par lesquelles s'exerce le pouvoir d'une organisation vont à la rencontre d'une implication (ou compliance) de l'individu qui non seulement partage 
les buts de l'organisation mais cherche à en améliorer les performances tout espérant en rester membre.

La notion même de transition professionnelle est sujette à variation : il a été démontré qu'elle ne saurait être réduite à un intervalle d'attente conduisant linéairement à un emploi en fonction des aléas du marché du travail, mais qu'elle est fortement encadrée par de multiples acteurs économiques et institutionnels, et qu'elle ne tend pas vers un modèle unique de parcours (Rose, 1998). Or, si les acteurs intermédiaires ont eu incontestablement leur mot à dire, le sens pris par les arrangements locaux a pu diverger de l'objectif initial couplant insertion des jeunes et émergence de besoins nouveaux. D'autre part, la diversité des parcours a été en partie « écrasée » par le modèle uniformisant du concours, réputé dans le milieu ainsi que dans la hiérarchie de l'institution comme la voie royale de l'installation professionnelle. Par différence, la dimension intégrative de la formation dispensée au ministère de l'Intérieur semble avoir mieux fonctionné, comme nous l'avons mentionné.

Enfin, sur le plan général des politiques d'emploi, ces difficultés rencontrées dans l'Éducation nationale, qui dispose pourtant de conditions comparativement favorables, n'incitent pas à disqualifier ce type de dispositif au profit de mesures privilégiant les ajustements de marché via l'allègement du coût du travail. $\mathrm{Au}$ contraire, elles invitent à baliser avec précision et avec une plus grande proximité territoriale les parcours suivis par les jeunes qui entrent dans le dispositif, en s'assurant de leur progressivité au fil des mois et des années.

\section{Bibliographie}

Alla L. (2000), «Acquisition et évaluation des compétences en situation scolaire », in Dolz J., Ollagnier E., L'énigme de la compétence en éducation, De Boeck Université, Bruxelles.

Argyris C. (1995), Savoir pour agir. Surmonter les obstacles à l'apprentissage organisationnel, InterEditions, Paris.

Barrère A. (2002), Enseignants au travail, L'Harmattan, Paris.

Becker H., Geer B., Hughes E., Strauss A. (1992) (1961) Boys in White. Students Culture in Medical School, New Brunswick and London, Transaction Publishers.

Bellier S. (1998), Le savoir-être dans l'entreprise, Vuibert, Paris.

Bellier S. (1999) «La compétence» in Carré P., Caspar P., Traité des sciences et des techniques de la formation, Dunod, Paris, pp. 223-244.
Bouclet M., Thomas D. (1999), Agir sur l'organisation du travail et la formation, ANACT, Éditions Liaisons, Rueil Malmaison.

Boudesseul G. (2003), « Considérer l'enseignement comme un travail : quelques implications de l'usage d'un concept pluridisciplinaire ", Cahiers de la MRSH, Caen, pp. 37-48.

Cadet J.-P., Diederichs-Diop L., Fournié D., Guitton C., Mahlaoui S. (2003), « Aides-éducateurs : à l'approche de l'échéance des premiers contrats, que sont devenues les fonctions ? Où en sont les jeunes? », Documents Evaluation Céreq, 170.

Cadet J.-P., Diederichs-Diop L., Fournié D. (2005a), «Organiser des transitions professionnalisantes pour les jeunes : l'exemple des aides-éducateurs », Formation Emploi - Revue Française de Sciences Sociales, 92, pp. 71-88.

Cadet J.-P., Diederichs-Diop L., Fournié D. (2005b), « Aides-éducateurs : à l'issue du dispositif emplois- 
jeunes, que reste-t-il des activités ? Que sont devenus les jeunes? », Relief Céreq, 10.

Chapoulie J.-M. (1987), Les professeurs de l'enseignement secondaire, un métier de classe moyenne, MSH, Paris.

Debost M. (2000), Audition de M. Debost, chargé de mission auprès du Directeur général de la Police Nationale - Groupe de Travail sur le bilan à miparcours des emplois-jeunes, Sénat, Rapports d'Information, www.senat.fr/rap/r00-025/r00-02531.html

Diederichs-Diop L., Fournié D. (2001), «École, collège, lycée : entre enseignement et éducation, les aides-éducateurs assurent de multiples activités », Éducation et Formations, 60.

Divay S. (éd.) (2003), «Regards croisés sur les emplois-jeunes », Documents Séminaire Céreq, 173.

Doyle W. (1985). "Recent research on classroom management : Implications for teacher preparation", Journal of Teacher Education, 36 (3), pp. 31-35.

Dubois N. (1994), La norme d'internalité et le libéralisme, Presses Universitaires de Grenoble.

Etzioni A. (1993), The Spirit of Community Rights, Responsibilities and the Communitarian Agenda, New York, Saint Martin's Press.

Fons J.-P., Meyer J.-L. (2005), «Les logiques de gestion de l'emploi public enseignant dans trois pays européens », Formation Emploi - Revue Française de Sciences Sociales, 92, pp. 5-20.

Hughes E. C. (1996) (1971), « Le travail et le soi », Le regard sociologique, Paris, EHESS, pp. 75-85.
Lessard C. (2000), «Présentation », in Education et Sociétés, «Nouvelles régulations et professions de l'éducation », 6,2, pp. 5-20.

Pagnani B. (2004), «Vers la reconnaissance des compétences construites in situ : un enjeu de professionnalisation pour les emplois-jeunes » in ChampyRemoussemard P., Meyer V., Etre emploi-jeune, et après?, Presses Universitaires de Nancy, pp. 127144.

Perrenoud P. (1999), Dix nouvelles compétences pour enseigner, Paris, ESF.

Piot T. (2001), «Aides-éducateurs : catalyseurs potentiels des transformations de l'école", Recherche et Formation, 37.

Piot T. (coord.) (2004), « Les aides-éducateurs dans les transformations de l'école», Les Sciences de l'Éducation. Pour l'Ere Nouvelle, 1.

Rose J. (1998), Les jeunes et l'emploi, Desclée de Brouwer, Paris.

Sainsaulieu R. (1977), L'identité au travail, Paris, FNSP.

Sennett R. (2000) (1998), Le travail sans qualités, Albin Michel, Paris.

Tardif M., Lessard J.-C. (1999), Le travail enseignant au quotidien, De Boëck Université, Bruxelles.

Zoyem J.-P. (2004), « Les nouveaux services-emplois jeunes. Bilan fin 2003 », Premières Synthèses, DARES, 20.1. 


\section{Résumé}

\section{Des aides-éducateurs « sans qualités »: cas limites ou révélateurs?}

\section{Gérard Boudesseul}

En quoi les contingences organisationnelles ordinaires et les relations professionnelles concrètes peuvent-elles hypothéquer un dispositif d'aide à l'insertion professionnelle qui accorde pourtant une grande importance à la construction de compétences? Plus largement, en quoi le rapport à la formation véhiculé dans une entreprise ou une institution peutil enclencher une dynamique fort lointaine des résultats espérés par tous? La difficulté de l'Éducation nationale à organiser une transition professionnelle pour les aides-éducateurs illustre ici un problème général rencontré, dès que l'on cherche à évaluer l'efficacité différentielle des politiques d'emplois aidés au regard des autres politiques d'emploi. Le récit de vie d'une douzaine d'aides-éducateurs montre comment un milieu d'accueil offrant des conditions a priori favorables à une reconnaissance de compétences nouvelles a pu contribuer à construire des jeunes salariés, et plus souvent des jeunes femmes, "sans qualités". Les notions même de transition professionnelle et de compétence sont alors en question.

\section{Mots clés}

Aide-éducateur, qualité de l'éducation, transition professionnelle, représentation de la formation, récit de vie.

Journal of Economic Literature : J 45, J 24 